\title{
Outcomes of atrial fibrillation ablation program based on single-shot techniques
}

\author{
Marek Jastrzębski ${ }^{1}$, Grzegorz Kiełbasa ${ }^{1}$, Kamil Fijorek², Adam Bednarski ${ }^{1}$, Aleksander Kusiak ${ }^{1}$, \\ Tomasz Sondej ${ }^{1}$, Agnieszka Bednarek ${ }^{1}$, Pawel Lis ${ }^{1}$, Agnieszka Olszanecka ${ }^{1}$, Marek Rajzer ${ }^{1}$
}

${ }^{1}$ First Department of Cardiology, Interventional Electrocardiology and Hypertension, Jagiellonian University Medical College, Krakow, Poland ${ }^{2}$ Department of Statistics, Cracow University of Economics, Krakow, Poland

Adv Interv Cardiol 2020; 16, 4 (62): 466-473

DOI: https://doi.org/10.5114/aic.2020.101773

\begin{abstract}
Introduction: Single-shot techniques such as cryoballoon and multipolar phased pulmonary vein ablation catheter (PVAC) are an alternative to the point-by-point radiofrequency method for atrial fibrillation (AF) ablation. However, there is a lack of data concerning sequential use of single-shot techniques, that is, for both the index and redo ablation.

Aim: To assess long-term outcomes of the 'single-shot techniques only' AF ablation strategy.

Material and methods: We analyzed all consecutive AF ablations performed over a 10-year period (2009-2019) in a center where a 'single-shot technique only' principle was followed from the start of the AF ablation program. Kaplan-Meier AF-free survival curves were calculated and complications were assessed on the basis of our prospectively maintained database.

Results: A total of 597 patients ( $62.4 \pm 12.5$ years) with paroxysmal (78.1\%) or persistent $(21.9 \%)$ AF entered the study and 655 AF ablation procedures were performed. In $96.5 \%$ of redos $(n=58)$ a different technique (mostly PVAC) was used than for the index ablation (mostly cryoballoon). The Kaplan-Meier estimates of 1,2 and 5 years freedom from AF were $78.2 \%, 69.2 \%$, and $56.0 \%$, for the index ablation, and $80.3 \%, 76.1 \%$ and $68.3 \%$, for the redo, respectively. The minor and major complication rates were $8.1 \%$, and $4.0 \%$, respectively.

Conclusions: An AF ablation program based solely on sequential use of two different single-shot techniques for both index and redo procedures is safe and effective. These observations might have important practical implications for new operators/centers starting AF ablation programs and for use of single-shot techniques for redo procedures.
\end{abstract}

Key words: atrial fibrillation ablation, redo ablation, single-shot technique, pulmonary vein ablation catheter, cryoballoon.

S u m m a ry

Long-term effectiveness and safety of an atrial fibrillation (AF) ablation program based on the principle of using only "single-shot" technique is reported. This novel AF ablation strategy was based on sequential use of two different single-shot techniques. Our results include, the first to date, assessment of efficacy of PVAC (pulmonary vein ablation catheter)-based ablation for AF redo procedures and suggest that various single-shot technique types can be successfully combined. These observations might have important practical implications for new operators/centers starting AF ablation programs and for use of single-shot techniques for redo procedures.

\section{Introduction}

Pulmonary vein (PV) isolation is a standard treatment option for patients with symptomatic atrial fibrillation (AF). Single-shot ablation techniques such as cryoballoon and multielectrode circular catheter emerged as a practical alternative to the classic point-by-point radiofrequency/3-dimensional system based AF ablation meth- od [1, 2]. Several studies have shown that outcomes of cryoballoon ablation, in terms of freedom from AF and safety profile, are comparable to point-by-point radiofrequency ablation $[3,4]$. Data concerning multielectrode phased radiofrequency ablation using pulmonary vein ablation catheter (PVAC, Medtronic) are less robust, although reported outcomes were similar [1]. However,

\section{Corresponding author:}

Prof. Marek Jastrzębski MD, PhD, First Department of Cardiology, Interventional Electrocardiology and Hypertension, Jagiellonian University Medical College, 2 Jakubowskiego St, 30-688 Krakow, Poland, phone: +48 502545 228, e-mail: mcjastrz@cyf-kr.edu.pl

Received: 7.10.2020, accepted: 26.10.2020. 
data concerning these two single-shot techniques came from centers/operators with substantial prior experience with radiofrequency/3-dimensional system based AF ablation. This might affect the single-shot technique outcomes, and the results of these studies might not be fully applicable to new centers or operators who want to rely solely on single-shot techniques. Moreover, there is a scarcity of data concerning the use of single-shot techniques for redo ablation when the index procedure was also performed with a single-shot technique, i.e. sequential use of two single-shot methods. Together these gaps in knowledge might lead to the underutilization of single-shot techniques.

\section{Aim}

The aim of this study was to investigate outcomes (AF-free survival, redo rate, redo efficacy and complications) of AF ablation in an electrophysiology laboratory where a 'single-shot technique only' principle was followed at the start of the AF ablation program, both for the index ablation and for all redo procedures.

\section{Material and methods}

\section{Study population}

This observational study enrolled consecutive patients with symptomatic paroxysmal, persistent and long-standing persistent $A F$, defined according to the Heart Rhythm Society (HRS)/European Heart Rhythm Association (EHRA) guidelines, with a history of failed treatment with one or more antiarrhythmic drugs (class I or III) [5]. On the basis of our prospectively maintained registry of all consecutive $\mathrm{PV}$ isolation procedures performed over a 10-year period, from June 2009 to September 2019 (to ensure a minimal follow-up of 6 months) we conducted a study analyzing outcomes of AF ablation after the index and redo single-shot technique based procedures. The study was approved by the institutional ethical committee of the Jagiellonian University (number 1072.6120.70.2020).

\section{Preprocedural management}

Transthoracic echocardiography using standard parasternal long and short axis views and apical two- and four-chamber views was performed within 1 week before ablation, enabling assessment of left ventricular ejection fraction and intracavitary dimensions. The anticoagulation strategy evolved during the study period. In the years 2009-2017 patients were anticoagulated with warfarin or acenocoumarol - with low-molecular weight heparin bridging before/after the procedure. Between 2017 and 2019, procedures were generally performed with uninterrupted oral anticoagulation (warfarin/acenocoumarol), while novel oral anticiagulatns were stopped shortly before CB ablation (morning dose skipped). The presence of left atrial thrombi was excluded by transesophageal echocardiography within $24 \mathrm{~h}$ before ablation in every patient. To exclude thrombus the left atrial appendage (LAA) was visualized in multiple two-dimensional TEE imaging planes. It was achieved by first developing the mid-esophageal aortic valve short-axis view $\left(30-60^{\circ}\right)$ and then anteflexing the transducer and rotating the multiplane angle from $0^{\circ}$ to $180^{\circ}$. Later, a multi-planar $\mathrm{LAA}$ reconstruction of $4 \mathrm{D}$ volume was prepared.

\section{Ablation procedure}

All procedures were performed by one of the two operators (M.J. or T.S.), both without any prior experience with $\mathrm{PV}$ isolation using the point-by-point radiofrequency technique. For the first ablation procedure, a cryoballoon was preferably used while for the redo procedures PVAC was favored. All ablation procedures were performed under conscious sedation (fentanyl and midazolam boluses). A single transseptal puncture using a Brockenbrough needle (BRK-1, St. Jude Medical, St Paul, MN, USA) and 8F transseptal sheath (SL-1, St. Jude Medical, St Paul, MN, USA) was performed under fluoroscopy using only contrast injection to confirm left atrial access. No prior PV anatomy assessment was performed; if considered necessary, PV anatomy was assessed with contrast/angiography during the procedure. His bundle catheter and coronary sinus catheter were used to facilitate transseptal puncture and also for phrenic nerve pacing and electrophysiological assessment of PV isolation. During the procedure intravenous heparin was administered to achieve an activated clotting time > $350 \mathrm{~s}$; measurements were performed every 30-40 min.

The 28-mm cryoballoon (Medtronic Cryocath, Minneapolis, MN, USA) was used in all patients. The procedure generally followed published expert consensus recommendations [6]. However, the cryoballoon technique evolved over the 10-year observation period of this study. Notably, the balloon design was changed from cryoballoon type 1 (CB1) to cryoballoon type 2 (CB2) to allow more uniform cooling and freeze protocols were appropriately modified; CB1 was used until November 2012. Optimal vein occlusion with total contrast retention, i.e. no back flow to the atrium, was aimed for; when this was not possible the 'pull-down' maneuver or overlapping applications were used. On the basis of published data freeze time was shortened from 5 mins to 3-4 min for left PVs and to 2-3 min for the right PVs and the number of applications was decreased from 3 per vein to 1-2 per vein. Both the freeze time and number of applications per vein were at the operator's discretion, guided by 'time to effect', minimal achieved temperature, thaw time, grade of the vein occlusion, use of 'pull-down' maneuver, the perceived arrhythmogenic potential of a particular vein, etc. For 226 consecutive patients ablated with CB2 the following information was gathered for better group 
characterization: number of cryoballoon applications per vein, total time of all cryoballoon applications per vein, minimal temperature achieved at 1 minute in each vein, minimal temperature at the end of cryoballoon application in each vein, PV entrance block time ("time-to-effect") in each vein.

The PVAC is a steerable, 9F, over-the-wire multielectrode circular mapping and ablation catheter that requires a multichannel radiofrequency (RF) generator (GENius; Medtronic, USA) in order to deliver synchronous duty-cycled phased unipolar and bipolar RF energy with its multiple electrodes. Overlapping 60-second RF applications were delivered in each PV. Ablation was mostly performed in a $2: 1$ bipolar/unipolar mode and switched to a $1: 1$ or $4: 1$ mode at the operator's discretion. We followed the ablation technique as described by others [1].

Regardless of the single-shot technique and/or protocol used for the energy application the procedural endpoint both for the index procedure and redo ablation was electrical isolation of all PVs. PV isolation was confirmed with the sudden disappearance of PV potentials or complete dissociation of PV potentials from atrial electrical activity; this was assessed in real time during cryoballoon freeze using an inner lumen circular multielectrode catheter (Promap, ProRhythm Inc., Ronkonkoma, NY, USA or Achieve, Medtronic Minneapolis, MN, USA). Alternatively, when disappearance of PV potentials was not visualized in real time during cryoenergy application, PV ostia were assessed for PV potentials and conduction block after the completion of a freeze-thaw cycle and this was compared with electrical activity at this site before cryoballoon application (template method). In case of recording suspicious electrical activity beyond the ablation line, pacing maneuvers from the coronary sinus, left atrial appendage and right atrium were performed to differentiate far-field signals from $P V$ potentials and also presence of exit block was assessed by pacing from the circular catheter within the PV. Because electrograms cannot be assessed during the delivery of phased RF current the template method combined with diagnostic pacing maneuvers was used during all PVAC-based ablation procedures. At the end of the procedure patients still in atrial fibrillation were electrically cardioverted, and when considered necessary PV isolation was re-assessed.

During ablation in the antra of the right-sided PVs, phrenic nerve function was monitored by continuous phrenic nerve stimulation in the superior vena cava combined with palpation of the upper abdomen in order to assess strength of diaphragmatic contraction. Moreover, in the last 307 patients a rule was adopted to stop cryoapplication when the freezing temperature dropped below $-56^{\circ} \mathrm{C}$.

\section{Post-ablation management}

Sheaths were usually removed $5 \mathrm{~h}$ after the procedure or, occasionally, immediately after the ablation, following protamine administration. Oral anticoagulation was started $6 \mathrm{~h}$ after sheath removal and continued for at least 3 months, and according to the CHADS2/ CHA2DS2-VASc scores thereafter. Preprocedural antiarrhythmic drugs were usually continued for 1-2 months. Some patients with long-persistent AF were treated with amiodarone for 3-6 months.

\section{Follow-up and study endpoints}

The analyzed primary endpoint of this study was freedom from AF recurrence. AF recurrence was defined as the first episode of AF lasting > $30 \mathrm{~s}$ diagnosed after the blanking period of 3 months. AF had to be documented by electrocardiogram (ECG), Holter monitoring or physician interpreted intracardiac electrogram from the implanted device (event marker data were considered unreliable). All patients were advised to have an ECG performed each time they experienced palpitations. Holter monitoring (24-72 h long) was scheduled after 3 and 6-9 months post-ablation and then advised once a year. Additionally, all patients received a telephone call at the time of conducting this study and were asked about symptoms, results of ECGs and Holter monitoring.

Secondary endpoint of this study was freedom from acute and long-term complications of AF ablation procedures. Acute (i.e. predischarge) complications were monitored and analyzed prospectively in accordance with the HRS/EHRA guidelines, published systematic reviews and other studies focused on specific complications of single-shot methods [7-12]. Long-term complications were determined on the basis of out-patient visits and/or final telephone conversation. Major complications were defined as any life-threatening situation or any adverse event with serious symptoms and/or permanent harm and/or requiring interventional treatment.

Following our strategy for redo procedures, a different single-shot technique was offered to the patients who had documented AF post-index ablation and remained symptomatic despite the reintroduction of $\beta$-blockers and/or class I antiarrhythmic drugs. The follow-up and endpoint assessment after a second PV isolation procedure was performed in the same way as after the index ablation.

\section{Statistical analysis}

Continuous variables are presented as means and standard deviations or medians and upper-lower quartile for data with non-normal distribution, while categorical variables are presented as numbers and percentages. For normality assessment the Shapiro-Wilk test was applied. The Kaplan-Meier method was used to estimate the survival functions for each endpoint. The log-rank test was used to compare survival distributions. Statistical analysis was performed in R 3.2. $P$-values $<0.05$ were considered statistically significant. 


\section{Results}

\section{Studied cohort and procedural characteristics}

We have identified 617 consecutive patients who underwent a single-shot technique based isolation of PVs in our center during the study period; of these, 20 cases were excluded for a number of reasons: prior point-bypoint PV ablation in other institution $(n=17)$, lack of follow-up data $(n=1)$, non-AF clinical arrhythmia $(n=1)$ or unrelated death $(n=1)$. Consequently, 597 patients, with a mean age of $57.9 \pm 10.6$ years, were analyzed with regard to both study endpoints. The majority of patients suffered from paroxysmal atrial fibrillation (78.1\%), were overweight/obese $(83.1 \%)$ and had some comorbidities (84\%); detailed baseline clinical characteristics of this cohort are presented in Table I. A total of 655 ablation procedures were performed. The median fluoroscopy time was 16.4 (11.2-25.0) min, median dose-area-product, re-

Table I. Baseline characteristics of the study population $(n=597)$

\begin{tabular}{|c|c|}
\hline \multicolumn{2}{|l|}{ Parameter } \\
\hline Age [years] & $57.9 \pm 10.6$ \\
\hline Male gender & $390(65.3 \%)$ \\
\hline BMI $\left[\mathrm{kg} / \mathrm{m}^{2}\right]$ & $29.1 \pm 4.3$ \\
\hline \multicolumn{2}{|l|}{ AF type: } \\
\hline Paroxysmal & $466(78.1 \%)$ \\
\hline Persistent & $105(17.6 \%)$ \\
\hline Long-standing persistent & $26(4.3 \%)$ \\
\hline Duration of AF history [months] & $36.0(18.0-72.0)^{\star}$ \\
\hline Number of failed antiarrhythmic drugs & $1.5 \pm 0.88$ \\
\hline Current/past smoker & $172(28.8 \%) / 38(6.2 \%)$ \\
\hline \multicolumn{2}{|l|}{ Comorbidities: } \\
\hline Hypertension & $397(66.5 \%)$ \\
\hline Diabetes mellitus & $76(12.7 \%)$ \\
\hline Chronic coronary syndrome & $60(10.1 \%)$ \\
\hline Heart failure & $27(4.5 \%)$ \\
\hline Structural heart disease ${ }^{\#}$ & $51(8.5 \%)$ \\
\hline Stroke/TIA & $59(9.9 \%)$ \\
\hline $\mathrm{CHA}_{2} \mathrm{DS}_{2}$-VASc score & $1.8 \pm 1.3$ \\
\hline $\mathrm{eGFR}<60 \mathrm{ml} / \mathrm{min} / 1.73 \mathrm{~m}^{2}$ & $63(10.5 \%)$ \\
\hline \multicolumn{2}{|l|}{ Echocardiography: } \\
\hline Left atrial diameter [mm] & $42.8 \pm 5.7$ \\
\hline Left ventricular ejection fraction, \% & $60.3 \pm 8.6$ \\
\hline $\begin{array}{l}\text { Left ventricular end-diastolic dimen- } \\
\text { sion }[\mathrm{mm}]\end{array}$ & $50.2 \pm 5.7$ \\
\hline
\end{tabular}

*Data presented as median and upper and lower quartile. "Defined as cardiomyopathy or artificial valve or severe valvular disease or left ventricular ejection fraction $<50 \%$. BMI - body mass index, AF - atrial fibrillation, TIA - transient ischemic attack, eGFR - estimated glomerular filtration rate. corded only for the last 267 cases, was 1885 (890-3826) $\mathrm{cG} \times \mathrm{cm}^{2}$. Acute success rate was $98 \%$; in $5(1.5 \%)$ patients one PV could not be isolated, in $2(0.5 \%)$ patients two PVs could not be isolated. Cryoballoon application characteristics (number of applications in each vein, minimal temperature etc.) for 226 non-selected, consecutive patients are presented in Table II.

\section{Index procedure outcomes}

For the index procedure cryoballoon $(n=589)$, or, much less frequently, PVAC $(n=8)$ ablation was performed. The 10-year observation period resulted in 520571 patient-days of follow-up. The median number of Holter ECG monitoring per patient was 3 (2-5) days; additionally 36 patients had data available from the implanted device capable of AF detection.

Table II. Characteristics of the cryotherapy dosage protocol $(n=226)$

\begin{tabular}{|c|c|}
\hline Parameter & Value \\
\hline \multicolumn{2}{|c|}{ Number of cryoballoon applications per vein, $n$ : } \\
\hline LSPV & $2.2 \pm 1.0$ \\
\hline LIPV & $1.8 \pm 0.7$ \\
\hline RSPV & $2.1 \pm 0.9$ \\
\hline RIPV & $2.0 \pm 0.8$ \\
\hline \multicolumn{2}{|c|}{ Duration of total freezing time per vein [s]: } \\
\hline LSPV & $351.9 \pm 143.6$ \\
\hline LIPV & $308.4 \pm 114.5$ \\
\hline RSPV & $243.7 \pm 101.0$ \\
\hline RIPV & $281.8 \pm 127.5$ \\
\hline \multicolumn{2}{|c|}{ Minimal temperature at $1 \min \left[{ }^{\circ} \mathrm{C}\right]$ : } \\
\hline LSPV & $41.8 \pm 4.4$ \\
\hline LIPV & $39.7 \pm 3.9$ \\
\hline RSPV & $43.1 \pm 4.6$ \\
\hline RIPV & $40.6 \pm 4.9$ \\
\hline \multicolumn{2}{|c|}{ Minimal temperature at the end of application $\left[{ }^{\circ} \mathrm{C}\right]$ : } \\
\hline LSPV & $49.0 \pm 5.3$ \\
\hline LIPV & $45.2 \pm 4.9$ \\
\hline RSPV & $48.9 \pm 5.1$ \\
\hline RIPV & $47.0 \pm 5.7$ \\
\hline \multicolumn{2}{|c|}{ Time-to-effect (PV entrance block) [s]: } \\
\hline LSPV $(61.1 \%)^{*}$ & $61.1 \pm 67.3$ \\
\hline LIPV $(41.6 \%)^{*}$ & $42.1 \pm 42.4$ \\
\hline $\operatorname{RSPV}(47.3 \%)^{*}$ & $43.4 \pm 24.8$ \\
\hline RIPV (29.6\%)* & $43.9 \pm 21.2$ \\
\hline
\end{tabular}

LSPV - left superior pulmonary vein, LIPV - left inferior pulmonary vein, RSPV right superior pulmonary vein, RIPV - right inferior pulmonary vein, $P V$ - pulmonary vein. ${ }^{*}$ Percentage of patients in whom it was possible to visualize real-time occurrence of pulmonary vein isolation. 


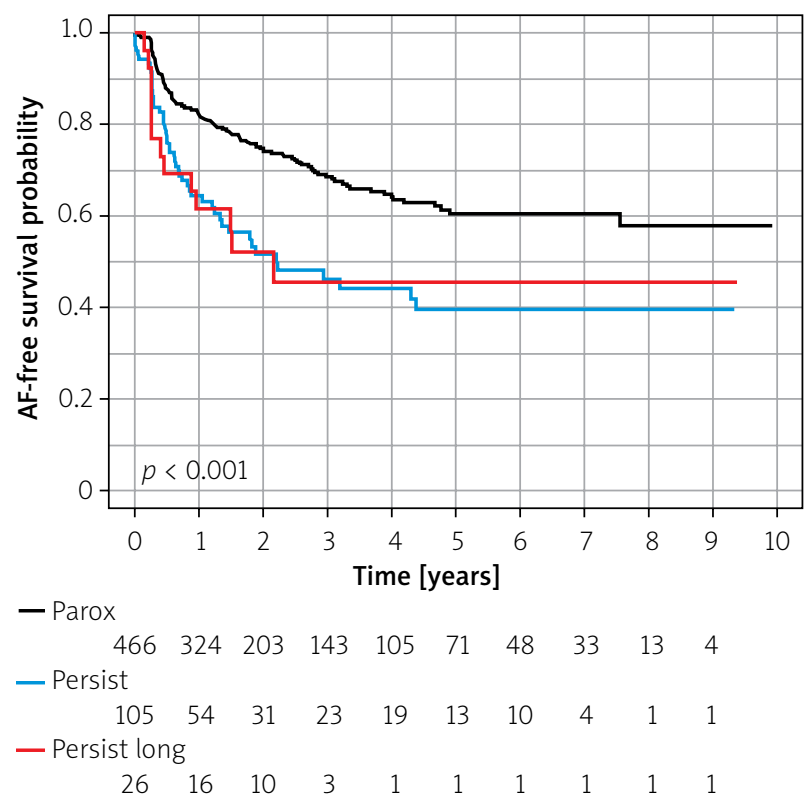

Figure 1. The Kaplan-Meier AF-free survival curve after the index AF ablation with regard to AF type

During the blanking period AF was observed in 73 (12.2\%) patients. The primary endpoint (AF recurrence, after the 3-month blanking period) was met in 197 (33.0\%) patients. The Kaplan-Meier estimates of 1-, 2- and 5-year freedom from $\mathrm{AF}$ after the index ablation were: $78.2 \%$ (95\% Cl: 74.9-81.6\%), 69.2\% (95\% Cl: 65.3-73.3\%), and $56.0 \%(95 \% \mathrm{Cl}: 50.9-61.6 \%)$, respectively. Success rate was higher for paroxysmal AF than for persistent/ long-standing persistent AF (Figure 1). The first 68 patients were treated with CB1, the rest with CB2. The Kaplan-Mei-

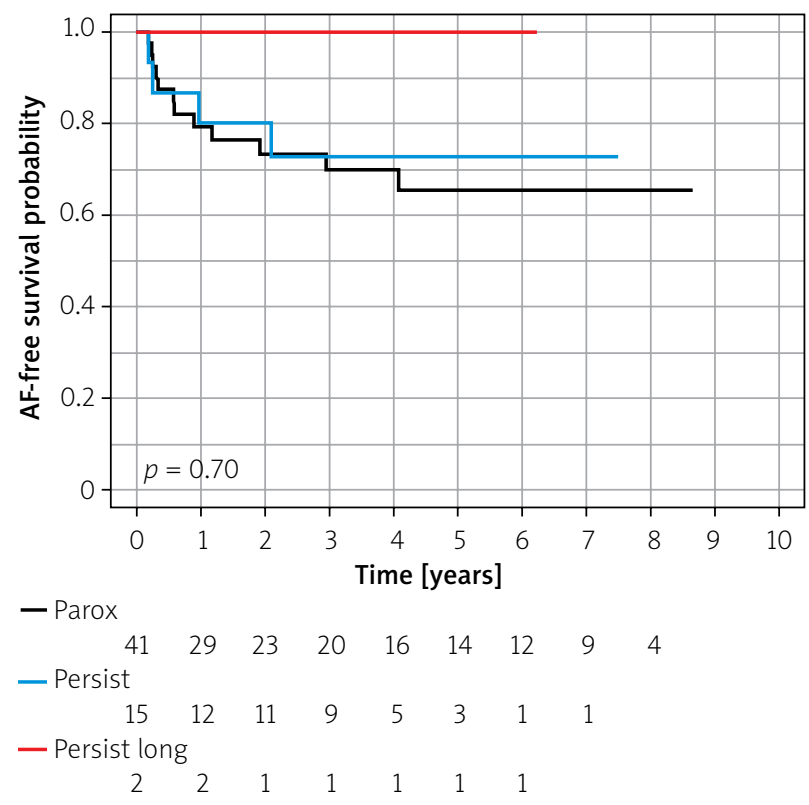

Figure 3. Kaplan-Meier AF-free survival curve after the redo ablation with regard to the AF type



Figure 2. The Kaplan-Meier AF-free survival curve after the index AF ablation with regard to type of cryoballoon used for ablation. CB1 - cryoballoon type 1 ; CB2 - cryoballoon type 2

er analysis of survival curves showed a 10\% lower longterm success rate when CB1 vs. CB2 was used (Figure 2).

\section{Redo procedure outcomes}

A total of 58 redo procedures were performed including 51 PVAC and 7 cryoballoon ablations; in $96.5 \%$ of cases a different single-shot technique was used for the second procedure. A median of 2 (1-3) PVs per patient showed reconnection; all PVs were successfully re-isolated. Redo patients were followed for 67.4 months (119 319 patient-days) after the re-ablation. AF recurrence after the 3-month blanking period was observed in 17 (29.3\%) patients. Kaplan-Meier estimates of 1-, 2- and 5-year freedom from AF after the redo procedure was $80.3 \%(95 \% \mathrm{Cl}$ : 70.4-91.4\%), 76.1 (95\% Cl: 65.5-88.4) and 68.3 (95\% Cl: 56.1-83.0), respectively; there was no difference in success rate between patients with paroxysmal AF vs. persistent/long-standing persistent AF (Figure 3).

\section{Post-ablation non-AF left atrial arrhythmias}

In 7 patients atypical atrial flutters were observed, 6 after index ablation and one after re-do ablation. In all but one of these patients concurrent AF recurrence was also noted. Since these arrhythmias are pathophysiologically distinct from AF they were treated with RF catheters rather than single-shot devices.

\section{Complications}

There were no deaths or permanent disabilities. In 72 (12.1\%) patients some complication occurred, including major complications in $4.0 \%$ of patients. Almost all complications occurred after the index ablation. In the redo procedures only one complication was noted - 
a persistent phrenic nerve paralysis. The most frequent complications were related to thermal phrenic nerve injury; these were closely followed by vascular access site complications and iatrogenic atrial septal defect requiring intervention (septal occluder implantation in 7 cases and cardiac surgery in 1 case). All complications, except one permanent phrenic nerve palsy, were resolved with appropriate medical treatment. A full list of observed complications is listed in Table III.

\section{Discussion}

This study showed the long-term effectiveness and safety of the 'single-shot technique only' ablation strategy for the management of AF in a large representative cohort of patients. The major findings were:

1) the sequential use of two different single-shot techniques is effective, resulting in freedom from AF at 2 years of $74 \%$ and $52 \%$, after the index procedure, and $73 \%$ and $80 \%$ after the redo procedure, for paroxysmal and persistent atrial fibrillation, respectively;

2) the PVAC-based method can be successfully used for redo AF ablation.

\section{AF-free survival after single-shot technique based ablation}

With each year of follow-up the percentage of AF-free patients falls, likely reflecting both a suboptimal result of the index ablation and the natural course/progression of the arrhythmia substrate. The reported AF recurrence rate at the second year after a single-shot technique based ablation is around $25-50 \%$ with moderate differences between different tools used (PVAC, cryoballoon, hot-balloon) and very comparable with the point-by-point radiofrequency current ablation method $[1-3,8,13]$. Our study provides a similar recurrence rate after a single procedure over the 10 years of follow-up (Kaplan-Meier survival curve in Figure 1). The novelty of our results lies in adding a new piece of data to the concept that the single-shot technique approach is completely equivalent and alternative to the classic point-by-point radiofrequency method, since in the current study such ablation outcomes were achieved using solely single-shot techniques and by operators who never used the classic point-by-point radiofrequency, 3D system based ablation method.

Additionally, the current study corroborates the data regarding higher efficacy of CB2 vs. CB1 [14], and expands it with very long-term follow-up data that show $10 \%$ absolute difference in AF recurrence rates after $5-6$ years (Figure 2).

Overall, $9.7 \%$ of patients underwent a redo during the study period; this constituted $29.4 \%$ of all patients with AF recurrence. The redo rate in our study was slightly lower than in most cryoballoon ablation studies that reported a 11.8-19.1 re-ablation rate [15-18], albeit similar to the rate of $7.8 \%$ obtained from the analysis of Swedish
Table III. Complications of single-shot technique based ablation observed in the current study

\begin{tabular}{|c|c|}
\hline Complications & $n(\%)$ \\
\hline \multicolumn{2}{|l|}{ Major: } \\
\hline Death related to the procedure & 0 \\
\hline Atrioesophageal fistula & 0 \\
\hline Symptomatic pulmonary vein stenosis & 0 \\
\hline Stroke & $1(0.17)$ \\
\hline Transient ischemic attack & $1(0.17)$ \\
\hline Tamponade/pericardiocentesis & $5(0.84)$ \\
\hline Tamponade requiring surgery & 0 \\
\hline Persistent phrenic nerve injury ${ }^{\S}$ & $7(1.17)$ \\
\hline Permanent phrenic nerve injury & $1(0.17)$ \\
\hline ASD requiring intervention & $8(1.34)$ \\
\hline Bleeding requiring blood transfusion & $1(0.17)$ \\
\hline \multicolumn{2}{|l|}{ Minor: } \\
\hline Vascular access site complications* & $9(1.51)$ \\
\hline Pericardial effusion & $4(0.67)$ \\
\hline Pericarditis & $1(0.17)$ \\
\hline Transient phrenic nerve injury" & $10(1.67)$ \\
\hline Migraine/headaches & $6(1.00)$ \\
\hline Hemoptysis & $2(0.33)$ \\
\hline Atelectasis/pneumonia & $5(0.84)$ \\
\hline Symptomatic gastroparesis & $2(0.33)$ \\
\hline Symptomatic inappropriate sinus tachycardia & $3(0.50)$ \\
\hline Other & $2(0.33)$ \\
\hline
\end{tabular}

ASD - atrial septal defect, spresent after discharge, *including large hematoma, arterio-venous fistula and pseudoaneurysm treated conservatively, and deep vein thrombosis; " resolved before discharge.

catheter ablation registry and the Atrial Fibrillation Ablation Long-Term registry of the European Heart Rhythm Association [4]. Our novel strategy for redo ablations, based on the principle that a different single-shot technique should be preferred for the second procedure, was effective, as freedom from AF after the second procedure was high (Figure 2). Such a strategy was employed based on the intuitive rationale that a failure of one single-shot technique based procedure might indicate that the left atrial anatomy was not suitable for the index single-shot technique and that using a different single-shot method has a higher chances of overcoming this limitation than using the same tools again. For example, a too large antrum might facilitate poor contact at some parts of the balloon perimeter and hence gaps that could be easily closed with PVAC using overlapping circular lesions while 
the balloon might fail again; a small antrum might not allow stabilization of the $28 \mathrm{~mm}$ balloon, or the PV takeoff angle or orifice shape might not allow good occlusion to be achieved or might result in off-axis cryoballoon to PV alignment, leading to poor contact of some segments with the balloon. When repeating ablation with balloon the same problems might repeat themselves, while with PVAC such anatomy might not constitute a problem. PVAC-based ablation after cryoballoon ablation for the index procedure was the dominant sequence employed by us. We are not aware of any study reporting outcomes of such an AF ablation strategy.

A limited number of studies have investigated outcomes of cryoballoon ablation for redo after the index procedure was also performed with a cryoballoon. Westra et al. studied 40 patients who had both the index and redo procedure performed with a cryoballoon and reported that at 1 year, $70 \%$ of patients were free of recurrent AF [16]. Similarly, Shade et al. used a cryoballoon for both the index and redo procedure in 47 patients and found that $60 \%$ of them had no AF recurrence during a 12-month follow-up period [17]. Cryoballoons were also used for redo procedures after the index ablation was performed with the point-by-point technique with 76.6-43\% of patients reported free from AF recurrence after 11-15 months of follow-up [19, 20]. Together, these reports suggest that cryoballoons are useful for re-ablation procedures; our data expand these observations to the other single-shot technique, namely, the PVAC. To the best of our knowledge there are no published data regarding the use of PVAC for redo procedures, suggesting that PVAC might be underused for this application.

\section{Complications of AF ablation based on single-shot techniques}

Several studies focusing on major complications of $\mathrm{AF}$ ablation using the point-by-point technique have reported complication rates between $3.9 \%$ and $4.9 \%$ [8, 9]. The major complication rate seen during ablation performed with cryoballoons, PVAC or other single-shot techniques seems to be similar or slightly lower, in the range of $2-6 \%[4,8,12,18,21]$. Our current study corroborates these observations and expands it to a setting with a more radical use of single-shot techniques. Of note, despite similar percentages, complication profiles of single-shot techniques are slightly different than complications of the point-by-point ablation method, and might, therefore, be more prone to reporting bias. Some complications such as significant iatrogenic atrial septal defect, gastroparesis or migraine headaches (observed together in $2.7 \%$ of patients in the current study) are not even mentioned in most papers focusing on complications of atrial fibrillation using the classic point-by-point radiofrequency method. Importantly, the single-shot technique based redo strategy used by us was not relat- ed to an increased percentage of complications; on the contrary, there was only a $2 \%$ rate of minor complications during redos. This stands in contrast to the pointby-point technique, where redo procedures were shown to be an independent predictor of major complications (odds ratio 3.6) [22].

\section{Limitations}

This was a single-center study with a small number of operators and related potential referral and treatment bias. Moreover, the ablation technique evolved and the equipment changed during the study; notably, after the initial 68 cases the CB1 was replaced by CB2, which resulted in a higher success rate. Due to the relatively small size of the CB1 cohort we believe that this was a minor limitation that we addressed in an additional Kaplan-Meier survival analysis. No long-term monitoring was performed in the majority of patients; therefore, the AF recurrence rate might have been underestimated. However, we believe that our monitoring approach reflected real life clinical practice.

\section{Conclusions}

This study suggests that a single-shot technique based ablation program results in similar effectiveness in atrial fibrillation ablation both for index and redo procedures and that the use of various single-shot technique types can be combined for a successful AF ablation program. These observations might have important practical implications for new operators/centers starting AF ablation programs and also warrant further studies to investigate the role of single-shot techniques for redo procedures.

\section{Conflict of interest}

The authors declare no conflict of interest.

\section{References}

1. Gal P, Aarntzen AE, Smit JJ, et al. Conventional radiofrequency catheter ablation compared to multi-electrode ablation for atrial fibrillation. Int J Cardiol 2014; 176: 891-5.

2. Neumann T, Vogt J, Schumacher B, et al. Circumferential pulmonary vein isolation with the cryoballoon technique results from a prospective 3-center study. J Am Coll Cardiol 2008; 52: 273-8.

3. Kuck KH, Brugada J, Furnkranz A, et al. Cryoballoon or radiofrequency ablation for paroxysmal atrial fibrillation. N Engl J Med 2016; 374: 2235-45.

4. Mortsell D, Arbelo E, Dagres N, et al. Cryoballoon vs. radiofrequency ablation for atrial fibrillation: a study of outcome and safety based on the ESC-EHRA atrial fibrillation ablation longterm registry and the Swedish catheter ablation registry. Europace 2019; 21: 581-9.

5. Calkins H, Hindricks G, Cappato R, et al. 2017 HRS/EHRA/ECAS/ APHRS/SOLAECE expert consensus statement on catheter and surgical ablation of atrial fibrillation: executive summary. Europace 2018; 20: 157-208. 
6. Su W, Kowal R, Kowalski M, et al. Best practice guide for cryoballoon ablation in atrial fibrillation: the compilation experience of more than 3000 procedures. Heart Rhythm 2015; 12: 1658-66.

7. Mugnai G, Sieira J, Ciconte G, et al. One year incidence of atrial septal defect after PV isolation: a comparison between conventional radiofrequency and cryoballoon ablation. Pacing Clin Electrophysiol 2015; 38: 1049-57.

8. Cappato R, Calkins H, Chen SA, et al. Worldwide survey on the methods, efficacy, and safety of catheter ablation for human atrial fibrillation. Circulation 2005; 111: 1100-5.

9. Steinbeck $G$, Sinner MF, Lutz M, et al. Incidence of complications related to catheter ablation of atrial fibrillation and atrial flutter: a nationwide in-hospital analysis of administrative data for Germany in 2014. Eur Heart J 2018; 39: 4020-9.

10. Aksu T, Golcuk S, Guler TE, et al. Gastroparesis as a complication of atrial fibrillation ablation. Am J Cardiol 2015; 116: 92-7.

11. Kato Y, Hayashi T, Kato R, Takao M. Migraine-like headache after transseptal puncture for catheter ablation: a case report and review of the literature. Intern Med 2019; 58: 2393-5.

12. Wasmer K, Foraita P, Leitz P, et al. Safety profile of multielectrode-phased radiofrequency pulmonary vein ablation catheter and irrigated radiofrequency catheter. Europace 2016; 18: 78-84.

13. Sohara H, Ohe T, Okumura K, et al. HotBalloon ablation of the pulmonary veins for paroxysmal AF: a multicenter randomized trial in Japan. J Am Coll Cardiol 2016; 68: 2747-57.

14. Conti S, Moltrasio M, Fassini G, et al. Comparison between firstand second-generation cryoballoon for paroxysmal atrial fibrillation ablation. Cardiol Res Pract 2016; 2016: 5106127.

15. Kuck KH, Albenque JP, Chun KJ, et al. Repeat ablation for atrial fibrillation recurrence post cryoballoon or radiofrequency ablation in the FIRE AND ICE trial. Circ Arrhythm Electrophysiol 2019; 12: e007247.

16. Westra SW, van Vugt SPG, Sezer S, et al. Second-generation cryoballoon ablation for recurrent atrial fibrillation after an index cryoballoon procedure: a staged strategy with variable balIoon size. J Interv Card Electrophysiol 2019; 54: 17-24.

17. Schade A, Langbein A, Spehl S, et al. Recurrence of paroxysmal atrial fibrillation after cryoisolation of the pulmonary veins. Is a "redo" procedure using the cryoballoon useful? J Interv Card Electrophysiol 2013; 36: 287-95.

18. Knight BP, Novak PG, Sangrigoli R, et al. Long-term outcomes after ablation for paroxysmal atrial fibrillation using the second-generation cryoballoon: final results from STOP AF Post-Approval Study. JACC Clin Electrophysiol 2019; 5: 306-14.

19. De Regibus, V, lacopino S, Abugattas JP, et al. Repeat procedures using the second-generation cryoballoon for recurrence of atrial fibrillation after initial ablation with conventional radiofrequency. J Interv Card Electrophysiol 2017; 49: 119-25.

20. Glowniak A, Tarkowski A, Fic P, et al. Second-generation cryoballoon ablation for recurrent atrial fibrillation after an index procedure with radiofrequency versus cryo: different pulmonary vein reconnection patterns but similar long-term outcome-Results of a multicenter analysis. J Cardiovasc Electrophysiol 2019; 30: 1005-102.

21. Mulder AA, Balt JC, Wijffels MC, et al. Safety of pulmonary vein isolation and left atrial complex fractionated atrial electrograms ablation for atrial fibrillation with phased radiofrequency energy and multi-electrode catheters. Europace 2012; 14: 1433-40.

22. Szegedi N, Szeplaki G, Herczeg S, et al. Repeat procedure is a new independent predictor of complications of atrial fibrillation ablation. Europace 2019; 21: 732-7. 